\title{
Evaluation of serum diagnosis of pancreatic cancer by using surface-enhanced laser desorption/ ionization time-of-flight mass spectrometry
}

\author{
HONGJUN GAO ${ }^{1 *}$, ZHAOXU ZHENG ${ }^{2 *}$, ZHIGANG YUE ${ }^{1}$, FANG LIU $^{3}$, \\ LANPING ZHOU $^{3}$ and XIAOHANG ZHAO 3,4
}

${ }^{1}$ Clinical Laboratory of Coal General Hospital; ${ }^{2}$ Department of Abdominal Surgery and ${ }^{3}$ State Key Laboratory of Molecular Oncology, Cancer Institute and Hospital, Chinese Academy of Medical Sciences and Peking Union Medical College; ${ }^{4}$ Center for Basic Medical Science, Navy General Hospital of Chinese PLA, Beijing, P.R. China

Received March 29, 2012; Accepted June 15, 2012

DOI: $10.3892 / \mathrm{ijmm} .2012 .1113$

\begin{abstract}
Proteomic methods have been widely used in disease marker discovery research. The aim of this study was to discover potential biomarkers for pancreatic cancer $(\mathrm{PCa})$ using surface-enhanced laser desorption/ionization time-offlight mass spectrometry (SELDI-TOF-MS). Crude serum samples from 132 patients with PCa and 67 healthy controls (HCs) were analyzed in duplicate using SELDI. Support vector machine (SVM) analysis of the spectra was used to generate a predictive algorithm based on proteins that were maximally differentially expressed between patients with $\mathrm{PCa}$ and the HCs in the training cohort. This algorithm was tested using leave-one-out cross-validation in the test cohort. From the 4 significant peaks in the training cohort, a classifier for separating patients with $\mathrm{PCa}$ from HCs was developed. The classifier was challenged with all samples achieving $96.67 \%$ sensitivity and $100 \%$ specificity in the training cohort and
\end{abstract}

Correspondence to: Professor XiaoHang Zhao, State Key Laboratory of Molecular Oncology, Cancer Institute and Hospital, Chinese Academy of Medical Sciences and Peking Union Medical College, Beijing 100021, P.R. China

E-mail: xhzdoctor@yahoo.cn

${ }^{*}$ Contributed equally

Abbreviations: PCa, pancreatic cancer; HCs, healthy controls; SVM, support vector machine; SELDI-TOF-MS, surface-enhanced laser desorption/ionization time-of-flight mass spectrometry; KNN, k-nearest neighbors; ANN, artificial neural network; WCX, weak cation exchange; DTT, dithiothreitol; SPA, sinapinic acid; $\mathrm{m} / \mathrm{z}$, massto-charge ratios; $\mathrm{CV}$, co-efficient of variance; $\mathrm{ROC}$, receiver operating characteristic; UDWT, undecimated discrete wavelet transform; QC, quality control; AUC, area under the curve; Da, dalton (one twelfth the mass of carbon-12)

Key words: pancreatic cancer, biomarker, crude serum, surfaceenhanced laser desorption/ionization time-of-flight, support vector machine
93.1\% sensitivity and $78.57 \%$ specificity in the test cohort. Additionally, the classifier correctly classified 12/12 stage Ia and 13/16 stage IIa PCa cases. The combination of the SELDI panel and CA19-9 was superior to CA19-9 alone in distinguishing individuals with $\mathrm{PCa}$ from the healthy subject group. These results suggest that high-throughput proteomic profiling has the capacity to provide new biomarkers for the early detection and diagnosis of PCa.

\section{Introduction}

Pancreatic cancer (PCa) is a uniformly lethal disease and is the 4th leading cause of cancer death in the USA with $>30,000$ estimated deaths per year. In recent years, the morbidity rate if $\mathrm{PCa}$ is gradually increasing in China. The majority of patients diagnosed with PCa succumb to the disease within months, and the 5 -year survival rate is only 3-4\% after diagnosis (1). Therefore, the diagnosis of PCa at an early stage or resectable phase is imperative in order to offer the best outcomes. The optimal approach for the early detection of PCa remains unknown. Molecular biomarkers may lead not only to the earlier diagnosis of pancreatic tumors but also to the more accurate diagnosis of these neoplasms (2). Unfortunately, tumor markers used for the auxiliary diagnosis of $\mathrm{PCa}$ in clinical practice do not have sufficient sensitivity and specificity to be applied to screening an asymptomatic population for the purpose of early detection. CA19-9, the widely used tumor marker for PCa, may be valuable for monitoring the therapeutic response of patients with PCa with an elevated serum CA19-9 level (3) and is considered the best test for PCa (4). However, approximately $10-15 \%$ of individuals can not secrete CA19-9 due to their negative Lewis antigen status. In addition, CA19-9 levels may be within the normal range while the tumor is small, asymptomatic and may be elevated in non-neoplastic conditions, such as hepatitis, benign biliary or pancreatic disease, greatly diminishing its specificity $(2,5)$. The sensitivity of CA19-9 is approximately $80 \%$, limiting its use for screening purposes, particularly for the diagnosis of resectable PCa (6). Since the majority of individuals with $\mathrm{PCa}$ have a poor prognosis, it is imperative to discover early detection strategies. 
Technologies applied in proteomics research, in particular surface-enhanced laser desorption/ionization time-of-flight mass spectrometry (SELDI-TOF-MS) and protein array techniques, are considered to be moving from research-focused applications to clinical laboratories as routine instruments for protein analysis (7). SELDI-TOF-MS profiling has the potential to be a safe and accurate diagnostic application in conjunction with conventional diagnostic methods for $\mathrm{PCa}(8)$. Although a number of possible serological tumor markers for PCa have been identified using the SELDI-TOF technique (9-14), few have been utilized as routine detection markers in oncological practice, and none have been thought to be valuable for PCa diagnosis. A previous study has reported that Chinese PCa patients may have different K-ras and p53 expressions from other populations (15). Different molecular characteristics may be concealed in serum profiling for a different racial population. Our study differs from previous reports as a larger number of early-stage PCa samples (stage Ia and $\mathrm{Ib}$ ) and a larger sample size were employed. The support vector machine (SVM) algorithm was used to analyze our raw data by comparing it with 2 other algorithms, the k-nearest neighbors (KNN) algorithm and the artificial neural network (ANN). Our study may provide a valuable clue for the early diagnosis of PCa.

\section{Materials and methods}

Sample collection and preparation. All samples were obtained from the Cancer Hospital of Peking Union Medical College (PUMC), Chinese Academy of Medical Sciences (CAMS), Beijing, China. The protocols of serum procurement, data management and blood collection were carried out as previously described (16). The Ethics Committee of the Cancer Hospital of PUMC and CAMS reviewed and approved our experimental procedures. Blood specimens from patients diagnosed with PCa were procured from the Department of Abdominal Surgery at the Cancer Hospital of PUMC. All healthy serum samples were obtained from volunteers for free clinic screening open to the general public. Only pretreatment samples obtained at the time of PCa diagnosis were used for this study. After obtaining informed consent from the patients, $\sim 3 \mathrm{ml}$ of whole blood were collected into a vacuum blood collection tube (red top tube, BD Biosciences) and centrifuged $1,000 \mathrm{x}$ g for $10 \mathrm{~min}$ at $4^{\circ} \mathrm{C}$, and the supernatant was transferred into a fresh $2 \mathrm{ml}$ EP tube and centrifuged $10,000 \times \mathrm{g}$ for $10 \mathrm{~min}$ at $4^{\circ} \mathrm{C}$. To avoid repeated freeze-thaw cycles, $50 \mu \mathrm{l}$ serum aliquots were refrozen at $-80^{\circ} \mathrm{C}$ until needed. These proceedings lasted for no more than $3 \mathrm{~h}$. A quality control (QC) sample was prepared by pooling an equal amount of serum from 50 specimens from age-matched healthy individuals. The QC sample was used to determine reproducibility and as a control protein profile for each SELDI experiment. A self-administered questionnaire was collected from each patient and included information such as gender, age, smoking and alcohol usage, as well as medical history. All sera were labeled with a unique number to protect the confidentiality of the patient. None of the samples were thawed more than twice before analysis.

The detailed characteristics of the healthy controls (HCs) and PCa patients are listed in Table I. The serum samples were divided into 2 groups to yield a training cohort comprising of 30 patients with pathological and (or) cytological evidence of PCa (CA19-9>300 U/ml) and $39 \mathrm{HCs}$ with no evidence of disease, as well as a test cohort comprising of 130 serum samples including $28 \mathrm{HCs}$ and $102 \mathrm{PCa}$ patients. We hypothesized that sera with a high CA19-9 value would possibly lead to the identification of potential cancer biomarkers. The clinical staging was based on the American Joint Committee on Cancer TNM classification of malignant cancers (version 6, published in 2002). A total of 28 early PCa (stage I) samples were employed in this study. The serum samples of other types of cancer of the digestive systemm, including 12 hepatocellular carcinomas (HCCs), 3 colorectal carcinomas and 6 gastric cancers were prepared for blinded validation with the pancreatic SELDI diagnostic pattern.

SELDI-TOF protein analysis. CM10 array (Ciphergen Biosystems, Fremont, CA), an advanced weak cation exchange (WCX) array, was used for serum protein profiling. All serum specimens were thawed in wet ice and then centrifuged at $10,000 \times \mathrm{g}$ for $2 \mathrm{~min}$. The supernatants were retained on ice immediately. A total of $10 \mu \mathrm{l}$ of U9 buffer [9 M urea, 2\% CHAPS, $1 \%$ dithiothreitol (DTT)] was added to $5 \mu \mathrm{l}$ of each serum sample in the 96-well cell culture plate, which was then agitated on a platform shaker at $600 \times \mathrm{g}$ at $4^{\circ} \mathrm{C}$ for $30 \mathrm{~min}$. CM10 chips were activated by adding $200 \mu 1$ of sodium acetate and agitated for 5 min twice. Next, $185 \mu \mathrm{l}$ of sodium acetate $(100 \mathrm{mM}, \mathrm{pH} 4)$ were added to the U9/serum mixture and the mixture was further agitated on a platform shaker for $2 \mathrm{~min}$. Diluted samples $(100 \mu \mathrm{l})$ were applied to each spot of protein chip immobilized on the bioprocessor (Ciphergen Biosystems). The bioprocessor was then sealed and agitated on a platform shaker for $1 \mathrm{~h}$ at $600 \mathrm{x} \mathrm{g}$ at $4^{\circ} \mathrm{C}$. The excess of serum mixtures was discarded. The chips were then washed 3 times with $200 \mu \mathrm{l}$ of sodium acetate and another 2 times with HPLC graded water. Finally, the chips were unloaded from the bioprocessor and air-dried. Prior to the SELDI-TOF-MS analysis, $1 \mu \mathrm{l}$ of saturated solution of $50 \%$ sinapinic acid (SPA) in $50 \%$ acetonitrile and $0.1 \%$ trifluoroacetic acid were applied onto each chip spot twice and the chips were air dried again.

Arrays were measured by a PBS II ProteinChip Reader (Ciphergen Biosystems) using an automated data collection protocol. The settings of the instrument were as follows: a high mass of mass-to-charge ratio $(\mathrm{m} / \mathrm{z}) 100,000$, an optimization range of $\mathrm{m} / \mathrm{z} 2,000-20,000$, a laser intensity of 180 units, a detector sensitivity of 7, a focus mass of $\mathrm{m} / \mathrm{z} 9,000$ (by optimization center) and a mass deflector of $\mathrm{m} / \mathrm{z} 1,000$. The SELDI acquisition parameters were set to $20, \Delta$ to 4 , transients/to 9 , and ending position to 80 . Mass accuracy was calibrated to $<0.1 \%$ with the all-in-one peptide molecular mass standard (Ciphergen Biosystems).

Bioinformatics and biostatistics. The mass spectra obtained from the spectrometer were first processed using Ciphergen ProteinChip software version 3.2.0 for baseline subtraction and automatic peak detection. Baseline subtraction is performed on a spectrum in isolation to eliminate any baseline signal that is caused mainly by chemical noise. Qualified mass peaks (signal-to-noise ratio $>4$ ) with $\mathrm{m} / \mathrm{z}$ between 2,000-20,000 were automatically detected. Peak clusters were 
Table I. Clinical characteristics of PCa patients and and healthy individuals.

\begin{tabular}{|c|c|c|c|c|c|c|c|c|}
\hline \multirow[b]{2}{*}{$\begin{array}{l}\text { Clinical } \\
\text { characteristics }\end{array}$} & \multicolumn{4}{|c|}{ Training set } & \multicolumn{4}{|c|}{ Test set } \\
\hline & No. & Age range & Mean age & $\begin{array}{l}\text { Falsely } \\
\text { classified }^{\text {a }}\end{array}$ & No. & Age range & Mean age & $\begin{array}{c}\text { Falsely } \\
\text { classified }^{\mathrm{a}}\end{array}$ \\
\hline \multicolumn{9}{|l|}{$\mathrm{HC}$ gender } \\
\hline Female & 16 & $25-70$ & 44.2 & 0 & 15 & $26-65$ & 42.2 & 2 \\
\hline Male & 23 & $35-77$ & 52.1 & 0 & 13 & $32-57$ & 40.5 & 4 \\
\hline \multicolumn{9}{|l|}{ PCa gender } \\
\hline Female & 12 & $42-65$ & 55.1 & 0 & 37 & $25-72$ & 55.5 & 2 \\
\hline Male & 18 & $43-75$ & 62.8 & 1 & 65 & $37-85$ & 59.1 & 5 \\
\hline \multicolumn{9}{|l|}{ PCa location } \\
\hline Bulb & 2 & $43-69$ & 56.0 & 0 & 21 & $34-73$ & 56.5 & 2 \\
\hline Cervix & 1 & 64 & 64.0 & 1 & 1 & 63 & 63.0 & 0 \\
\hline Cervix and body & 5 & $50-69$ & 58.4 & 0 & 3 & $51-68$ & 61.0 & 0 \\
\hline Body & 0 & 0 & 0 & 0 & 3 & $39-62$ & 51.3 & 0 \\
\hline Body and tail & 4 & $42-75$ & 59.5 & 0 & 15 & $25-82$ & 60.5 & 1 \\
\hline Head & 18 & $46-75$ & 60.3 & 0 & 59 & $37-85$ & 57.6 & 4 \\
\hline \multicolumn{9}{|l|}{ PCa diagnosis } \\
\hline Pathology & 21 & $42-75$ & 59.4 & 1 & 46 & $34-82$ & 55.8 & 6 \\
\hline Cytology & 15 & $53-75$ & 61.5 & 0 & 26 & $25-85$ & 55.4 & 1 \\
\hline Clinic & 0 & 0 & 0 & 0 & 36 & $37-73$ & 59.3 & 0 \\
\hline \multicolumn{9}{|l|}{ PCa pathological types } \\
\hline Duct adenocarcinoma & 28 & $42-75$ & 59.1 & 1 & 59 & $25-85$ & 56.9 & 6 \\
\hline Cystadenocarcinoma & 0 & 0 & 0 & 0 & 1 & 71 & 71.0 & \\
\hline Signet-ring cell carcinoma & 1 & 75 & 75.0 & 0 & 0 & 0 & 0 & 0 \\
\hline Mucinous adenocarcinoma & 1 & 62 & 62.0 & 0 & 0 & 0 & 0 & 0 \\
\hline Adenosquamous carcinoma & 0 & 0 & 0 & 0 & 5 & $37-75$ & 52.0 & 0 \\
\hline Carcinoma sarcomatodes & 0 & 0 & 0 & 0 & 1 & 56 & 56.0 & 1 \\
\hline Unclear & 0 & 0 & 0 & 0 & 36 & $37-73$ & 59.3 & 0 \\
\hline \multicolumn{9}{|l|}{ PCa pathological differentiation } \\
\hline Well & 1 & 62 & 62.0 & 0 & 5 & $44-68$ & 56.4 & 1 \\
\hline Well and moderate & 0 & 0 & 0 & 0 & 5 & 43-77 & 62.6 & 0 \\
\hline Moderate & 6 & $42-69$ & 55.2 & 1 & 18 & $37-75$ & 54.3 & 2 \\
\hline Moderate and poor & 1 & 61 & 61.0 & 0 & 7 & $34-70$ & 53.6 & 0 \\
\hline Poor-differentiated & 5 & $49-61$ & 58.0 & 0 & 6 & $25-73$ & 48.5 & 1 \\
\hline Not reported & 17 & $46-75$ & 61.6 & 0 & 25 & $37-85$ & 60.0 & 3 \\
\hline \multicolumn{9}{|l|}{ PCa staging } \\
\hline Ia & 0 & 0 & 0 & 0 & 12 & $37-77$ & 55.7 & 0 \\
\hline $\mathrm{Ib}$ & 0 & 0 & 0 & 0 & 16 & $25-74$ & 52.6 & 3 \\
\hline IIa & 0 & 0 & 0 & 0 & 16 & $42-85$ & 60.3 & 2 \\
\hline $\mathrm{IIb}$ & 10 & $43-69$ & 58.2 & 1 & 6 & $49-71$ & 60.0 & 0 \\
\hline III & 13 & $50-75$ & 61.5 & 0 & 33 & $37-82$ & 57.6 & 1 \\
\hline IV & 7 & $42-75$ & 58.7 & 0 & 19 & $39-73$ & 60.9 & 1 \\
\hline
\end{tabular}

${ }^{a}$ No. of patients that were falsely classified by the surface-enhanced laser desorption/ionization (SELDI) pattern. HC, healthy control; PCa, pancreatic cancer.

completed with second-pass peak selection (signal-to-noise ratio $>2$, within a $0.3 \%$ mass window), and estimated peaks were added. The peak intensities were normalized to the total ion current of $\mathrm{m} / \mathrm{z}$ between 2,000-20,000. The co-efficient of variance $(\mathrm{CV})$ of the $\mathrm{QC}$ serum was calculated using the Biomarker Wizard software package. 
Univariate analysis was performed between the groups using the Wilcoxon test and the results were considered significantly different when the P-value was $<0.05$. For each putative marker and panel, receiver operating characteristic (ROC) curves were generated to evaluate their discriminatory power.

To discriminate between patients with PCa and healthy individuals, the diagnosis pattern was established with the following procedure: the raw data analysis was performed by the Zhejiang University, Cancer Institute-ProteinChip Data Analysis System (ZUCI-PDAS) (www.zlzx.net) (17). Briefly, after obtaining the registered account number, we uploaded our raw data to the ZUCI-PDAS server in the '.xml' format transformed by the ProteinChip software for each profiling, and the 'sample.txt' and 'group.txt' files arranged according to the requirements were uploaded. In this process, it was necessary to emphasize that the uploaded data were the raw data without baseline correction, as a previous study indicated that the baseline correction prevented reproduction of their initial results (18). The data were first analyzed using the undecimated discrete wavelet transform (UDWT) method to denoise the signals (19). The spectra were subjected to baseline correction by aligning with a monotone local minimum curve and mass calibration by adjusting the intensity and mass/ charge scale according to 3 labeled peaks that appear in all the selected spectra. The parameters were designated as follows: the top 10 significant peaks were selected; the signal-to-noise ratio was not $<4$; the algorithm was SVM; the minimal percentage of each peak appearing in all spectra was 10 and the mass size window was not $>0.3 \%$. The leave-one-out cross-validation approach was applied in order to estimate the accuracy of this classifier. The SVM model with the highest Youden's index was selected as the model for detecting pancreatic carcinoma and the remote server was then run. For the final step, the spectrum data in the test set were updated onto the server in the 'xml' format, and the named 'Trainfact' and 'TestFact' files generated in the training procedure were uploaded. The training and test results were downloaded online when the analysis was completed. All the procedures of the panel construction and validation for the new test set were performed conveniently by the ZUCI-PDAS.

In order to evaluate which algorithm was more suitable to our data, we compared the performance of SVM, KNN and ANN using ROC curves. The ROC curves were generated with the ROCR package of the R-project free available software version 2.3.1 (20) (www.r-project.org).

\section{Results}

$Q C$ and reproducibility. The $\mathrm{QC}$ serum sample, 50 pooled sera from healthy individuals, was used to determine reproducibility and as a control protein profile for each SELDI experiment. QC spectra selected from the course of the analysis, were used to calculate the $\mathrm{CV}$ of intensity and mass/charge (mass drift). The range of intensity $\mathrm{CV}$ for the 25 selected peak heights was from $12.6-30.2 \%$ and the mean was $20 \%$; the range of mass/ charge CV was from $0.04-0.0797 \%$ and the mean was $0.05 \%$ (data not shown).

Peak detection and evaluation of candidate diagnosis pattern In the training cohort, a total of 105 significant peaks $(\mathrm{P}<0.01)$
Table II. Statistics of the 4 candidate biomarkers used to discriminate pancreatic cancer from healthy individuals.

\begin{tabular}{cccc}
\hline $\mathrm{m} / \mathrm{z}$ & P-value & $\begin{array}{c}\text { HCs } \\
\text { mean } \pm \mathrm{SD}\end{array}$ & $\begin{array}{c}\text { PCa } \\
\text { mean } \pm \mathrm{SD}\end{array}$ \\
\hline 7775 & $1.3 \mathrm{E}-09$ & $539.94 \pm 1107.18$ & $3054.52 \pm 2054.35$ \\
8567 & $4.8 \mathrm{E}-09$ & $9757.83 \pm 3687.34$ & $3393.56 \pm 2790.46$ \\
5362 & $2.16 \mathrm{E}-08$ & $313.91 \pm 109.22$ & $762.29 \pm 379.51$ \\
5344 & $9.08 \mathrm{E}-08$ & $970.23 \pm 1210.87$ & $4319.07 \pm 3077.86$ \\
\hline
\end{tabular}

HC, healthy controls; PCa, pancreatic cancer; m/z, mass-to-charge ratio.

were found using the Wilcoxon test after noise filtering, normalization, alignment and peak cluster detection. These peaks were ranked according to the P-value of the Wilcoxon rank sum test. The P-values of the top 10 significant peaks were all $<10^{-8}$. The top ten peaks were $4390,8773,7775,8567$, 5362, 4141, 4080, 4289, 5344 and 8661 dalton (Da). The SVM analyzed the sensitivity and specificity of the random combination of these peaks as a classifier to discriminate between $\mathrm{PCa}$ and $\mathrm{HC}$ samples. The peak combination with the highest accuracy was selected as the candidate biomarker diagnosis pattern. Finally, 7775, 8567, 5362 and 5344 Da formed the potential biomarker pattern. The peaks of 7775,5362 and $5344 \mathrm{Da}$ were all upregulated and the peak of $8567 \mathrm{Da}$ was downregulated in PCa patients (Fig. 1). Overlay spectra of the 4 peaks $(7775,8567,5362$ and $5344 \mathrm{Da})$ for the 30 patients and 39 healthy individuals are displayed in Fig. 2. There are different levels overlapping in each peak between the PCa and $\mathrm{HC}$ groups. The statistical results including the $\mathrm{P}$-value and the means \pm standard deviation (SD) of the 4 peaks in the PCa and HC groups are shown in Table II. The classifier had a 100\% specificity and $96.67 \%$ sensitivity for diagnosis of the training set itself through the leave-one-out cross validation. This classifier discriminated cancer patients from healthy individuals in the test cohort with a sensitivity of $93.1 \%$ (95 of 102) and a specificity of $78.57 \%$ (22 of 28 ). For the different stages of PCa, $100 \%$ (12/12) of stage Ia, $81.2 \%$ (13/16) of stage Ib, $87.5 \%$ $(14 / 16)$ of stage IIa, $100 \%$ (6/6) of stage IIb, $96.97 \%$ (32/33) of stage III and $94.74 \%$ (18/19) of stage IV cases were correctly classified by the classifier. For early-stage $\mathrm{PCa}$, the sensitivity of CA19-9 (cut-off value, $37 \mathrm{U} / \mathrm{ml}$ ) was $83.3 \%$ (10/12) for stage Ia and $68.8 \%$ (11/16) for stage Ib. The power of each peak and the diagnostic pattern in discriminating between patients with $\mathrm{PCa}$ from healthy individuals was determined by estimating the area under the ROC curve (AUC). The ROC and AUC values of each peak and their combination in the 69 cases of the training cohort are shown in Fig. 3. The SELDI panel (m/z 7775, 8567, 5362 and 5344) was more efficient than CA19-9 in distinguishing individuals with $\mathrm{PCa}$ from the healthy subject groups $(\mathrm{P}<0.05)$. The sensitivity of the SELDI panel was $93.1 \%$ but that of CA19-9 was only $72.5 \%$ in the test cohort. Combining the SELDI protein peaks and CA19-9 yielded a significant improvement for CA19-9 at distinguishing between serum from patients with PCa and that of HCs. The sensitivity of the combination of the SELDI panel and CA19-9 was $97.1 \%$ in the test cohort and $97.7 \%$ in the total patient 

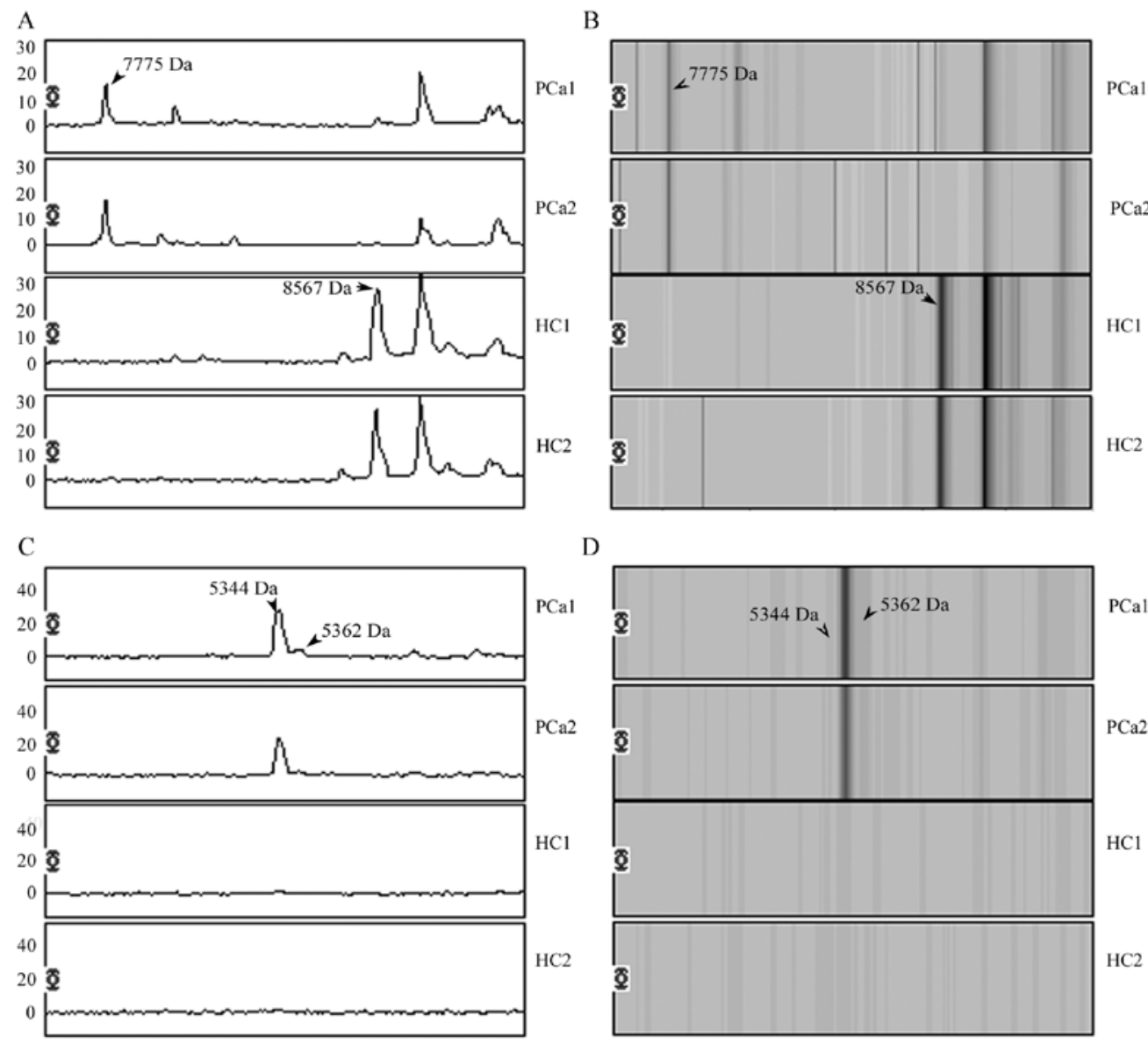

Figure 1. Representative examples of the SELDI spectral and gel-view of healthy control (HC) and pancreatic cancer (PCa) patients are shown with the molecular weight calculation (m/z values) along the $\mathrm{x}$-axis and relative intensity along the $\mathrm{y}$-axis. PC1-2 and HC1-2 were 2 representative samples of PCa and healthy individuals, respectively. The peaks of 7775, 5344 and 5362 were upregulated and 8567 was downregulated in the PCa group.

Table III. Detailed evaluation of the sensitivity of the SELDI diagnostic pattern and its combination with CA19-9.

\begin{tabular}{|c|c|c|c|c|c|c|c|c|}
\hline \multirow[b]{2}{*}{ Stage } & \multicolumn{4}{|c|}{ Test cohort (\%) } & \multicolumn{4}{|c|}{ Test and training cohorts (\%) } \\
\hline & $\mathrm{n}$ & CA19-9 & Pattern & Combination & $\mathrm{n}$ & CA19-9 & Pattern & Combination \\
\hline Ia & 12 & $10(83.3)$ & $12(100)$ & $12(100)$ & 12 & $10(83.3)$ & $12(100)$ & $12(100)$ \\
\hline $\mathrm{Ib}$ & 16 & $11(68.8)$ & $13(81.3)$ & $14(87.5)$ & 16 & $11(68.8)$ & $13(81.3)$ & $14(87.5)$ \\
\hline IIa & 16 & $13(81.3)$ & $14(87.5)$ & $16(100)$ & 16 & $13(81.3)$ & $14(87.5)$ & $16(100)$ \\
\hline $\mathrm{IIb}$ & 6 & $4(66.7)$ & $6(100)$ & $6(100)$ & 16 & $14(87.5)$ & $15(93.8)$ & $16(100)$ \\
\hline III & 33 & $23(69.7)$ & $32(97)$ & $32(97)$ & 46 & $36(78.3)$ & 45 (97.8) & $45(97.8)$ \\
\hline IV & 19 & $13(68.4)$ & $18(94.7)$ & $18(94.7)$ & 26 & $20(76.9)$ & $25(96.2)$ & $25(96.2)$ \\
\hline Total & 102 & 74 (72.6) & $95(93)$ & $99(97.1)$ & 132 & $104(78.8)$ & $124(93.9)$ & $129(97.7)$ \\
\hline
\end{tabular}

population (Table III). The SVM as a powerful classification tool has been widely applied in bioinformatics $(21,22)$. In the present study, the SVM learning algorithm was optimized to analyze our data by comparing the distinguishing ability of SVM to that of the KNN and the artificial nerve net (ANN) (Fig. 4). In our study, we also included patients with other types of gut cancer in order to challenge the PCa classifier. Consequently, $58.3 \%$ (7/12) of HCCs, 33.3\% (1/3) of colon cancer and $66.7 \%(4 / 6)$ of gastric cancer cases were correctly classified as cancer. These results suggest that the 4 peaks may be specifically associated with PCa but not with other types of gut cancer.

\section{Discussion}

We employed SELDI-TOF-MS technology to uncover the discriminating information hidden in the proteome of $\mathrm{PCa}$ patient serum. Zinkin et al (23) showed that SELDI-TOF-MS could accurately distinguish patients with HCC from those with hepatitis $C$ virus cirrhosis and was more accurate than 

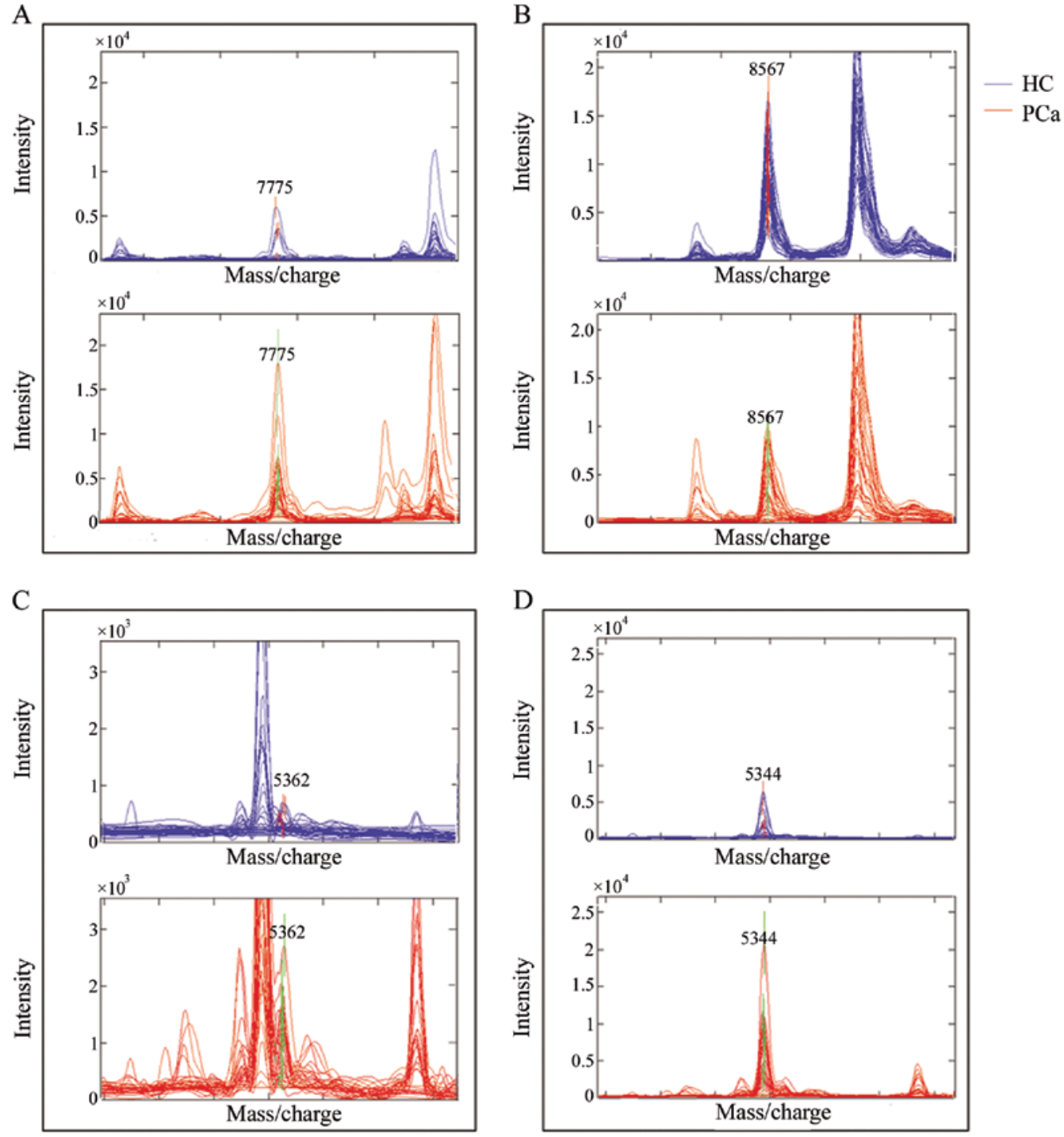

Figure 2. Overlay of the surface-enhanced laser desorption/ionization (SELDI) spectra of the 4 selected peaks by the panel displayed in the healthy control (HC) and pancreatic cancer (PCa) groups. A-D represent the peaks 7775, 8567, 5362 and 5344, respectively. The blue and red curves indicate the HC and PCa groups, respectively.

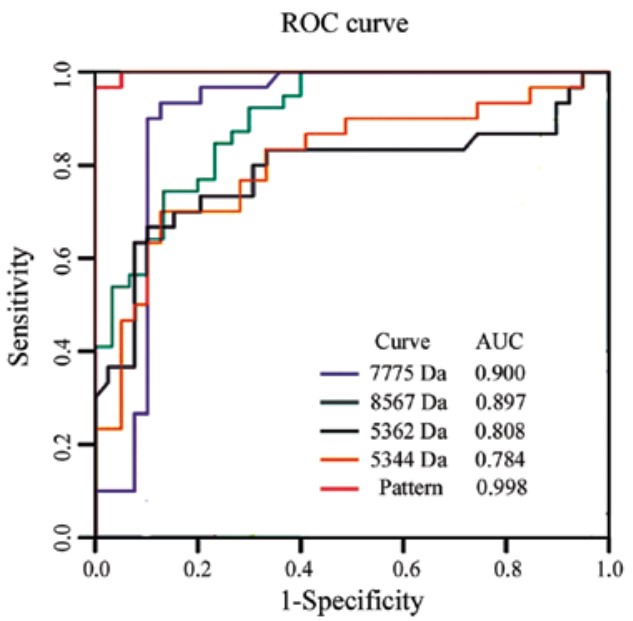

Figure 3. Receiver-operator-characteristics (ROC) curve for the performance of each peak involved in panels and surface-enhanced laser desorption/ionization (SELDI)-derived marker panels. AUC values of 0.9 for the $\mathrm{m} / \mathrm{z} 7775$ peak (purple line), 0.897 for the $\mathrm{m} / \mathrm{z} 8567$ peak (green line), 0.808 for the $\mathrm{m} / \mathrm{z} 5362$ peak (black line), 0.784 for the $\mathrm{m} / \mathrm{z} 5344$ peak (orange line) and 0.998 for the pattern (peach line).

\section{SVM, KNN and ANN for prediction}

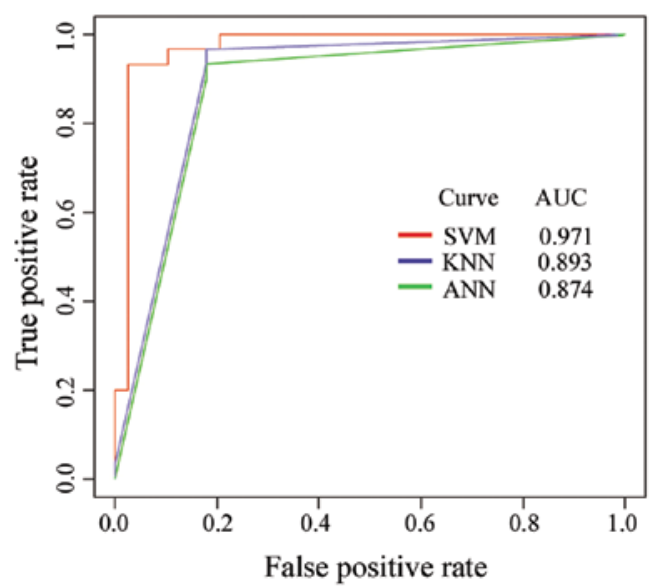

Figure 4. Receiver-operator-characteristics (ROC) curve for the performance of the 3 algorithms support vector machine (SVM), k-nearest neighbors (KNN) and artificial neural network (ANN). The area under the curve (AUC) value of 0.971 for the SVM (red line), 0.893 for the KNN (blue line) and 0.874 for the ANN (green line). 
traditional biomarkers in identifying small tumors. A number of studies have reported that PCa serum, plasma, pancreatic juice or tissue profiling may be used to discover tumor markers for discriminating PCa samples from controls using SELDI-TOF-MS coupled with various protein chips including IMAC $(9-11,13,24), \operatorname{WCX}(10), \operatorname{H} 50(8,12)$, CM10 $(12,13)$ and Q10 (25). A number of studies have conducted similar experiments using stepwise anion exchange chromatography prior to using the ProteinChip system $(9,10,12,13)$. Zinkin et al (23) suggested that whole serum, rather than serum depleted of high abundant proteins, be used for SELDI measurements, as within the range of molecular weights of proteins detected by SELDI-TOF, the depletion of albumin and other high abundant proteins does not dramatically change the pattern and level of low molecular weight proteins detected by SELDI-TOF. In addition, the reproducibility of the whole process with processed serum was worse than that with crude serum (12), and certain potential protein may be lost. Therefore, the fraction steps of serum or plasma prior to identifying biomarkers using the SELDI-TOF technology may not be optimal. Expanding the study to encompass other populations and strategies will lead to the discovery of additional potential biomarkers. In this study, crude serum samples from 132 patients with $\mathrm{PCa}$ and 67 healthy individuals of Han nationality were used for protein-chip analysis, allowing a higher throughput and improving reproducibility. To the best of our knowledge, we used the highest number of PCa cases for the largest for the serological biomarkers study using SELDI-TOF-MS. Another different characteristic in this research was that there were more early-stage PCa samples (28 cases of stage I) used for the SELDI-TOF evaluation. The final characteristic of the experimental design differing from previous studies was that the case number of patients with PCa in the test set was far greater than that in the training set. For the limited cases with $\mathrm{PCa}$, the sensitivity of the classifier received greater credibility when additional cases were used to validate the SELDI pattern.

The mean CV of the intensity of the peaks existing in all the QC serum profiling at different spots was $20 \%$ (range, 12.2-30.2\%), which was comparable with the CVs reported by other groups (10-43\%) for SELDI serum profiling (26-33). The mean CV of $\mathrm{m} / \mathrm{z}$ of peaks was $0.05 \%$ (range, $0.04-0.08 \%$ ) which was similar to the CVs reported by previous studies $(27,29,34)$. Proteomic profiling of the serum from the training cohort was evaluated by the other 2 algorithms, KNN and ANN. The AUC suggested that SVM was a better algorithm for our data.

Using the CM10 ProteinChip array, we identified groups of PCa-associated proteins (biomarker protein panels) significantly expressed in patients with PCa. Four proteins of mass 7775, 8567, 5362 and 5344 Da were selected as biomarkers to correctly discriminate between patients with $\mathrm{PCa}$ and the healthy individuals. The peak of $8567 \mathrm{Da}$, a promising isolated mass, was of particular interest as it was decreased in the patients with PCa and was also identified by 2 other similar separate studies using the WCX and H50 chip, respectively $(8,10)$ (likely the same protein/peptide). Tumor-derived proteins secreted into the extracellular spatial or bloodstream were usually expected to be mined in the study of serum samples from cancer patients. Although the decreased expression or loss of serum peaks as classifiers in cancer patients is different from the serum biomarkers currently used in clinics, the similar biomarker was found in gastric cancer (35). The mass of $7775 \mathrm{Da}$ was also identified in another study, a serum profiling study of esophageal squamous cell carcinoma (unpublished data). We confirmed all the case archives of the samples that failed to be classified by the SELDI pattern, and found that 1 patient with benign adenoma was incorrectly sorted to the duct adenocarcinoma group. The perfect example illustrated that it was possible for the panel to be developed in order to discern between benign and malignant pancreatic lesions, although more benign cases needed to be validated. Due to the relatively low prevalence of PCa, an increased sensitivity is required for the early detection of $\mathrm{PCa}$ in the asymptomatic population (9). The SELDI pattern in this study had a high sensitivity of 96.67 and $93.1 \%$ in the training and test cohorts, respectively. The predictive capacity of the mass peaks identified in this study require further testing, including the examination of a larger panel of serum from patients with PCa of stage I, various other malignancies and benign diseases.

SELDI-TOF-MS is currently the most widely used and advertised non gel-based method. However, little is known about the potential of this technique for future application. Many different peaks have been purified and identified using various methods, as summarized by Hortin (36). All these proteins are non tumor-specific and most are derived from host non-specific response. Although still preliminary and requiring validation on an independent dataset, these results provide incentive to further explore SELDI-based serum proteomics as a prognostic and/or predictive tool. Schwegler et al (38) maintained that the profile itself was diagnostic, and extending its use does not depend on the identification of the proteins in discriminating peaks in the emerging field of MS-based protein profiling of body fluids (37). We deem that it is immature to identify a SELDI-TOF panel as the sensitivity and recovery rate of the fraction strategy and identification technology is far less compared to the need for low abundance significant proteins.

Our results provide the premise for further evaluation and validation of this SELDI proteomic classification system for the early detection and diagnosis of PCa. However, further study is required to construct profiles for the identification of PCa. Our findings are in general agreement with those reported by previous studies, thus providing additional confirmation that a proteomic approach may accurately identify clinical PCa. Various purification and serial efforts to identify the low-mass protein biomarkers discovered in this study are currently ongoing.

\section{Acknowledgements}

This study was supported by the National Natural Science Foundation of China (nos. 30572126 and 30721001), the High-Tech R\&D Program of China (nos. 2006AA02Z19B, 2006AA02Z341 and 2006AA02A403) and the National Basic Research Program of China (no. 2004CB518707). We thank Professor Shu Zheng from the Second Affiliated Hospital of Zhejing University for helpful suggestions and technical support in this study. The authors also thank Dr Jie-kai Yu for his assistance in data analysis. 


\section{References}

1. Li D, Xie K, Wolff R and Abbruzzese JL: Pancreatic cancer Lancet 363: 1049-1057, 2004.

2. Yeo TP, Hruban RH, Leach SD, et al: Pancreatic cancer. Curr Probl Cancer 26: 176-275, 2002.

3. Abrams RA, Grochow LB, Chakravarthy A, et al: Intensified adjuvant therapy for pancreatic and periampullary adenocarcinoma: survival results and observations regarding patterns of failure, radiotherapy dose and CA19-9 levels. Int J Radiat Oncol Biol Phys 44: 1039-1046, 1999.

4. Pleskow DK, Berger HJ, Gyves J, Allen E, McLean A and Podolsky DK: Evaluation of a serologic marker, CA19-9, in the diagnosis of pancreatic cancer. Ann Intern Med 110: 704-709, 1989.

5. Akdogan M, Sasmaz N, Kayhan B, Biyikoglu I, Disibeyaz S and Sahin B: Extraordinarily elevated CA19-9 in benign conditions: a case report and review of the literature. Tumori 87: 337-339, 2001.

6. Ni XG, Bai XF, Mao YL, et al: The clinical value of serum CEA, CA19-9, and CA242 in the diagnosis and prognosis of pancreatic cancer. Eur J Surg Oncol 31: 164-169, 2005.

7. Petricoin E III and Liotta LA: Counterpoint: The vision for a new diagnostic paradigm. Clin Chem 49: 1276-1278, 2003.

8. Scarlett CJ, Smith RC, Saxby A, et al: Proteomic classification of pancreatic adenocarcinoma tissue using protein chip technology. Gastroenterology 130: 1670-1678, 2006.

9. Bhattacharyya S, Siegel ER, Petersen GM, Chari ST, Suva LJ and Haun RS: Diagnosis of pancreatic cancer using serum proteomic profiling. Neoplasia 6: 674-686, 2004.

10. Koopmann J, Zhang Z, White N, et al: Serum diagnosis of pancreatic adenocarcinoma using surface-enhanced laser desorption and ionization mass spectrometry. Clin Cancer Res 10: 860-868, 2004

11. Yu Y, Chen S, Wang LS, et al: Prediction of pancreatic cancer by serum biomarkers using surface-enhanced laser desorption/ ionization-based decision tree classification. Oncology 68: 79-86, 2005.

12. Honda K, Hayashida Y, Umaki T, et al: Possible detection of pancreatic cancer by plasma protein profiling. Cancer Res 65: 10613-10622, 2005.

13. Ehmann M, Felix K, Hartmann D, et al: Identification of potential markers for the detection of pancreatic cancer through comparative serum protein expression profiling. Pancreas 34: 205-214, 2007.

14. Song J, Patel M, Rosenzweig CN, et al: Quantification of fragments of human serum inter-alpha-trypsin inhibitor heavy chain 4 by a surface-enhanced laser desorption/ionization-based immunoassay. Clin Chem 52: 1045-1053, 2006.

15. Dong M, Nio Y, Tamura K, et al: Ki-ras point mutation and p53 expression in human pancreatic cancer: a comparative study among Chinese, Japanese, and Western patients. Cancer Epidemiol Biomarkers Prev 9: 279-284, 2000.

16. He P, He HZ, Dai J, et al: The human plasma proteome: analysis of Chinese serum using shotgun strategy. Proteomics 5: 3442-3453, 2005.

17. Wang JX, Yu JK, Wang L, Liu QL, Zhang J and Zheng S: Application of serum protein fingerprint in diagnosis of papillary thyroid carcinoma. Proteomics 6: 5344-5349, 2006.

18. Baggerly KA, Morris JS and Coombes KR: Reproducibility of SELDI-TOF protein patterns in serum: comparing datasets from different experiments. Bioinformatics 20: 777-785, 2004.

19. Braun S, Vogl FD, Naume B, et al: A pooled analysis of bone marrow micrometastasis in breast cancer. N Engl J Med 353 793-802, 2005.

20. Sing T, Sander O, Beerenwinkel $N$ and Lengauer T: ROCR: visualizing classifier performance in R. Bioinformatics 21 3940-3941, 2005.
21. Pavlidis P, Wapinski I and Noble WS: Support vector machine classification on the web. Bioinformatics 20: 586-587, 2004.

22. Wagner M, Naik DN, Pothen A, et al: Computational protein biomarker prediction: a case study for prostate cancer. BMC Bioinformatics 5: 26, 2004.

23. Zinkin NT, Grall F, Bhaskar K, et al: Serum proteomics and biomarkers in hepatocellular carcinoma and chronic liver disease. Clin Cancer Res 14: 470-477, 2008.

24. Rosty C, Christa L, Kuzdzal S, et al: Identification of hepatocarcinoma-intestine-pancreas/pancreatitis-associated protein I as a biomarker for pancreatic ductal adenocarcinoma by protein biochip technology. Cancer Res 62: 1868-1875, 2002.

25. Melle C, Ernst G, Escher N, et al: Protein profiling of microdissected pancreas carcinoma and identification of HSP27 as a potential serum marker. Clin Chem 53: 629-635, 2007.

26. Petricoin EF, Ardekani AM, Hitt BA, et al: Use of proteomic patterns in serum to identify ovarian cancer. Lancet 359 : 572-577, 2002.

27. Adam BL, Qu Y, Davis JW, et al: Serum protein fingerprinting coupled with a pattern-matching algorithm distinguishes prostate cancer from benign prostate hyperplasia and healthy men. Cancer Res 62: 3609-3614, 2002.

28. Rai AJ, Stemmer PM, Zhang Z, et al: Analysis of Human Proteome Organization Plasma Proteome Project (HUPO PPP) reference specimens using surface enhanced laser desorption/ ionization-time of flight (SELDI-TOF) mass spectrometry: multi-institution correlation of spectra and identification of biomarkers. Proteomics 5: 3467-3474, 2005.

29. Semmes OJ, Feng Z, Adam BL, et al: Evaluation of serum protein profiling by surface-enhanced laser desorption/ionization time-of-flight mass spectrometry for the detection of prostate cancer: I. Assessment of platform reproducibility. Clin Chem 51: 102-112, 2005.

30. Aivado M, Spentzos D, Alterovitz G, et al: Optimization and evaluation of surface-enhanced laser desorption/ionization time-of-flight mass spectrometry (SELDI-TOF MS) with reversed-phase protein arrays for protein profiling. Clin Chem Lab Med 43: 133-140, 2005.

31. Ward DG, Cheng Y, N'Kontchou G, et al: Changes in the serum proteome associated with the development of hepatocellular carcinoma in hepatitis C-related cirrhosis. Br J Cancer 94: 287-292, 2006

32. Albrethsen J, Bogebo R, Olsen J, Raskov H and Gammeltoft S: Preanalytical and analytical variation of surface-enhanced laser desorption-ionization time-of-flight mass spectrometry of human serum. Clin Chem Lab Med 44: 1243-1252, 2006.

33. Ward DG, Cheng Y, N'Kontchou G, et al: Preclinical and posttreatment changes in the HCC-associated serum proteome. $\mathrm{Br} \mathrm{J}$ Cancer 95: 1379-1383, 2006.

34. Roboz J: Mass spectrometry in diagnostic oncoproteomics. Cancer Invest 23: 465-478, 2005.

35. Ebert MP, Meuer J, Wiemer JC, et al: Identification of gastric cancer patients by serum protein profiling. J Proteome Res 3: 1261-1266, 2004

36. Hortin GL: The MALDI-TOF mass spectrometric view of the plasma proteome and peptidome. Clin Chem 52: 1223-1237, 2006.

37. Petricoin EF and Liotta LA: SELDI-TOF-based serum proteomic pattern diagnostics for early detection of cancer. Curr Opin Biotechnol 15: 24-30, 2004.

38. Schwegler EE, Cazares L, Steel LF, et al: SELDI-TOF MS profiling of serum for detection of the progression of chronic hepatitis $\mathrm{C}$ to hepatocellular carcinoma. Hepatology 41: 634-642, 2005. 